\title{
ALCOOLISMO: UM NOVO DESAFIO PARA O ENFERMEIRO
}

\author{
Alcoholism: a new Chalenge for the nurse \\ Alcoholismo: un nuevo desafío para el enfermero
}

Laura Acauan $^{1}$

Marilurde Donato ${ }^{2}$

Ana Maria Domingos ${ }^{3}$

\section{RESUMO}

Levantamento da produção científica sobre alcoolismo entre 1986 e 2006, com enfoque em Psiquiatria e Saúde Pública. Fontes bibliográficas: livros, revistas científicas, dissertações, teses e publicações do Centro Brasileiro de Informações sobre Drogas Psicotrópicas (CEBRID). Utilizou-se a palavra "alcoolismo" para a consulta, via Internet, na base de dados do CEBRID e da Biblioteca Virtual em Saúde. Pesquisou-se também nas bibliotecas do Instituto de Psiquiatria, da Faculdade de Farmácia e da Escola de Enfermagem Anna Nery, Unidades da Universidade Federal do Rio de Janeiro. Identificaram-se 62 publicações sobre 0 assunto, sendo utilizadas apenas 14. As restantes foram excluídas por não correlacionarem o tema àquelas áreas. Nove publicações tiveram enfoque em Saúde Pública, três em Psiquiatria e duas em ambas as áreas. As autoras concluíram que a produção foi incipiente, cabendo ao enfermeiro buscar novos saberes acerca do alcoolismo para prestar uma assistência eficiente e eficaz a esta clientela específica.

Palavras-chave: Alcoolismo. Enfermagem. Saúde Pública. Psiquiatria.

\begin{abstract}
Mapping of the scientific production about alcoholism between 1986 and 2006, focusing on Psychiatry and Public Health. Bibliographic sources: books, magazines, scientific magazines, Dissertations, Thesis and publications of the Centro Brasileiro de Informações sobre Drogas Psicotrópicas (CEBRID) - Brazilian Center of Informations about Psychotropic Drugs. The word "alcoholism" has been used for consultation, by Internet, on the data base of CEBRID and the Virtual Library on Health. A research was made as well on the libraries of the Psychiatric Institute, of the Pharmacy College and the Nursing College Anna Nery, units of the Universidade Federal do Rio de Janeiro. Sixty-two publications about the subject were identified but only fourteen were used. The rest was excluded because it was not related to the subject on these domains. Nine publications focused on Public Health, three on Psychiatrics and two on both domains. The authors concluded that the production was incipient; the nurses have to search for new knowledge about the alcoholism to offer an efficient assistance to these specific clients.
\end{abstract}

Keywords: Alcoholism. Nursing. Public Health. Psychiatrics.

\section{Resumen}

Relevamiento de la producción científica sobre alcoholismo entre 1986 y 2006, con enfoque en Psiquiatría y Salud Pública. Fuentes bibliográficas: libros, revistas científicas, Disertaciones, Tesis y publicaciones del Centro Brasileño de Informaciones sobre Drogas Psicotrópicas - Centro Brasileiro de Informações sobre Drogas Psicotrópicas (CEBRID). Se ha utilizado la palabra "alcoholismo" para consultación, vía Internet, en la base de datos del CEBRID y de la Biblioteca Virtual en Salud. Se ha investigado también en las bibliotecas del Instituto de Psiquiatría, de la Facultad de Farmacia y de la Escuela de Enfermería Anna Nery, unidades de la Universidad Federal de Río de Janeiro. Se han identificado sesenta y dos publicaciones sobre el tema, siendo que se utilizaron apenas catorce. Las restantes han sido excluidas por no relacionarse al tema de aquellas áreas. Nueve publicaciones tuvieron enfoque en Salud Pública, tres en Psiquiatría y dos en ambas áreas. Las autoras concluyeron que la producción fue incipiente, cabiéndole al enfermero buscar nuevos saberes acerca del alcoholismo para prestar una asistencia eficiente y eficaz a esta clientela específica. 


\section{CONSIDERAÇÕES INICIAIS}

0 alcoolismo é considerado um dos mais graves problemas de saúde pública em todo o mundo. Tem como principal característica, segundo a Organização Mundial de Saúde (OMS), o estado físico e psíquico resultante da ingestão de álcool, com o surgimento de reações comportamentais que incluem a compulsão pela ingestão contínua ou periódica da substância, cuja finalidade é experimentar os efeitos psíquicos causados pela bebida e evitar o desconforto ocasionado pela sua falta.

Não há "um protótipo de alcoolista" ${ }^{2}$, assim como já está confirmado que 0 alcoolismo "não se restringe a uma etnia, estado civil, emprego ou escolaridade". ${ }^{2} 0$ que se sabe é que o consumo abusivo de álcool e seus efeitos decorrem de inúmeros fatores, dentre os quais destacam-se alguns "propiciadores do maior consumo de álcool"3, a exemplo do excesso de propaganda na mídia em geral, estimulando o surgimento do vício e incentivando o consumo a qualquer preço ${ }^{4}$, e também do grande número de trabalhadores não-qualificados existente no país, submetidos a uma alta rotatividade empregatícia que lhes causaria "desesperança" e "sentimento de solidão" ${ }^{3}$ decorrentes de uma precária situação sócioeconômica, fazendo com que recorressem ao uso abusivo de álcool, o que confirmaria as evidências de que o alcoolismo é "bastante prevalente em nossa população de baixa renda"."

0 consumo alcoólico excessivo tem acarretado graves problemas, com conseqüências familiares, profissionais e sociais para os indivíduos e a coletividade. ${ }^{5}$ Isto ocorre por se tratar de substância considerada lícita e aceita pela sociedade, com o agravante de que seu uso também é estimulado pela falta de fiscalização e inobservância das restrições legais para a sua venda.

0 uso crônico do álcool causa alterações comportamentais (agressividade,conflitos familiares, violência urbana e doméstica) e comprometimentos orgânicos (p. ex., hipertensão arterial, gastrite, cirrose) e clínicos (p. ex., depressão, doenças mentais), que são as causas para buscar cuidados de saúde 6 , contribuindo também para a alta prevalência de acidentes automobilísticos e 0 absenteísmo laboral. ${ }^{7}$ Conseqüentemente, é um problema que onera os cofres públicos, pelos prejuízos que causa à sociedade. ${ }^{7,4}$

Como substância psicoativa, o álcool produz efeito depressor ou euforizante, e seu consumo não pode ser entendido como um fenômeno marginal, já que $10 \%$ dos residentes nos centros urbanos, independente de sexo, idade, nível de escolaridade e poder aquisitivo, consomem álcool livremente, mesmo sabendo da repercussão dos seus efeitos orgânicos, psicológicos, familiares e sociais. ${ }^{8}$

No que diz respeito aos prejuízos orgânicos, vale lembrar que o álcool é uma substância que fornece calorias vazias de proteínas, vitaminas e sais minerais, diminui o sintoma da fome, mas piora a desnutrição, não só por propiciar uma menor ingestão de alimentos, como também por alterar a digestão e absorção do ingerido, bem como a ativação das vitaminas a nível hepático. ${ }^{3,9}$
0 abuso da bebida é considerado um dos dez comportamentos de maior risco à saúde, causando a morte de 1,8 milhões de pessoas no mundo; destas, $5 \%$ representam jovens entre 15 e 29 anos de idade, confirmando que as pessoas ainda estão longe de ter uma relação equilibrada com esta substância. ${ }^{10}$

0 alcoolismo vem despertando a atenção do poder público e dos profissionais da saúde pelo seu potencial patogênico e alta prevalência de casos que podem culminar em internações que "pouco contribuem para modificar o hábito alcoólico"3 às vezes servindo apenas "como fonte de repouso e alimentação por grande parte da população de alcoólatras".3

Todavia, deve-se considerar que, em grande parte dos casos de internação, "as equipes de saúde não estão devidamente capacitadas para identificar e tratar a doença, em especial levando em conta que o diagnóstico precoce é a chave para a prevenção das complicações de saúde dela advindas"2, e também por ser considerada uma síndrome "com natureza progressiva e fatal". ${ }^{4}$

$\mathrm{Na}$ área da saúde, um dos maiores grupos é o de profissionais de enfermagem. Por ser também aquele que passa a maior parte do tempo junto aos usuários dos serviços de saúde, têm melhores condições de auxiliar os que apresentam problemas relacionados com a ingestão abusiva de álcool. Para tanto, é fundamental que o resgate da história de consumo de substâncias psicoativas se torne uma rotina nas avaliações dos enfermeiros, em qualquer instituição de saúde, pública ou privada, já que muitas são as lacunas de conhecimento em relação ao cuidado de uma clientela historicamente excluída e isolada do convívio da sociedade. ${ }^{6}$

Mas, "a pouca informação educacional, ou a falta dela, leva à deficiência nos cuidados prestados, a atitudes negativas, percepções estereotipadas e pessimistas com relação aos bebedores-problema e ao insucesso do tratamento". ${ }^{6} \mathrm{~A}$ literatura consultada referiu que "os enfermeiros tendem a ser mais moralistas com relação aos alcoolistas e percebem 0 problema mais como fraqueza do que como doença; também são mais moralistas se comparados com outros profissionais de saúde". ${ }^{6,}{ }^{11} \mathrm{E}$, ainda, que a percepção do enfermeiro com relação ao alcoolismo "tem sido mais no enfoque de doença do que moral", por isso "acreditam que essa população deve receber assistência de saúde como outro doente qualquer"6,12. Essa visão "preconceituosa que as pessoas geralmente têm sobre o alcoolista, deixa entrever uma ambigüidade diante da questão, visto que reconhecem a necessidade de tratamento do indivíduo que abusa da bebida alcoólica". ${ }^{4}$

Assim sendo, é imprescindível que estes profissionais detenham conhecimentos específicos "para problemas físicos e psicológicos" 6 acerca desta problemática, a fim de que um número maior de pacientes tenha a oportunidade de receber intervenção e tratamento adequados em relação à dependência alcoólica.

Quanto ao papel da equipe de enfermagem junto ao alcoolista, é importante lembrar que, no Brasil, a partir da criação da Lei $n^{\circ} 775 / 49$, os currículos de graduação em 
enfermagem passaram a prever a formação de profissionais voltados para atuar na área de Psiquiatria, cuja prática assistencial sempre foi influenciada diretamente pelo saber médico construído sobre o doente mental, tanto que "cabia às enfermeiras apenas vigiar, acompanhar, reprimir e controlar os doentes mentais ou alienados, como eram designados no século $X X "{ }^{13}$

Este perfil manteve-se durante muito tempo, sendo as enfermeiras percebidas como meras ajudantes dos médicos psiquiatras por atuarem sempre sob suas ordens, além de serem consideradas mal preparadas para prestar cuidados ao doente mental, em face da ausência de um saber científico próprio. ${ }^{13}$

Deve-se ressaltar, entretanto, que a procura pela capacitação profissional neste campo de atuação é incipiente, ainda mais quando relacionada à dependência alcoólica, o que prejudica a formação dos enfermeiros, alguns dos quais não se sentem preparados para enfrentar uma clientela com problemas tão específicos. Quanto à área de Enfermagem de Saúde Pública, esta capacitação é ainda mais escassa ${ }^{14}$, o que prejudica sobremaneira as ações de promoção e prevenção levadas a efeito por instituições de saúde públicas ou privadas.

A desinformação e o aparente desinteresse demonstrado por muitos profissionais de enfermagem em relação aos problemas decorrentes do uso abusivo do álcool foram percebidos durante a pesquisa dos textos oriundos do levantamento bibliográfico, o que ensejou a elaboração deste artigo.

\section{METODOLOGIA}

Este estudo constituiu-se numa revisão da produção científica nacional, com o intuito de identificar textos sobre alcoolismo, elaborados por profissionais de saúde, com enfoque em Psiquiatria e/ou Saúde Pública, no período de 1980 a 2006.

Foram utilizados como fontes bibliográficas: livros, revistas científicas, dissertações, teses e publicações do Centro Brasileiro de Informações sobre Drogas Psicotrópicas (CEBRID).

0 trabalho foi realizado em duas etapas: a primeira, via Internet, na base de dados do CEBRID e da Biblioteca Virtual em Saúde (BVS); e a segunda, nas bibliotecas do Instituto de Psiquiatria (IPUB), da Faculdade de Farmácia (FF) e da Escola de Enfermagem Anna Nery (EEAN), Unidades pertencentes à Universidade Federal do Rio de Janeiro (UFRJ).

Dentre as referências consultadas e selecionadas eletronicamente, algumas foram adquiridas a partir das fontes, enquanto outras foram impressas pelas pesquisadoras, por estarem disponíveis na íntegra, naqueles portais da Internet.

O levantamento realizado, a partir da palavra "alcoolismo", em periódicos nacionais permitiu identificar 62 publicações que abordavam o assunto genericamente, mas apenas 14 foram utilizadas nesta revisão, isto porque as outras 48 foram excluídas por não deixarem evidente o encaminhamento do tema para as áreas anteriormente citadas.

A leitura criteriosa do conteúdo da produção científica obtida permitiu chegar aos resultados apresentados nos quadros a seguir.

\section{RESULTADOS}

Quadro 1 - Produção científica sobre alcoolismo na década de 1980 - Rio de Janeiro, 2007

\begin{tabular}{|c|c|c|c|c|}
\hline $\begin{array}{c}\text { Ano de } \\
\text { Publicação }\end{array}$ & Título & Autor(es) & $\begin{array}{c}\text { Abordagem } \\
*\end{array}$ & Edição \\
\hline 1982 & $\begin{array}{l}0 \text { uso e abuso do } \\
\text { álcool como } \\
\text { problema de } \\
\text { saúde pública }\end{array}$ & $\begin{array}{c}\text { FREITAS } \\
\mathrm{J}\end{array}$ & $\mathrm{SP}$ & $\begin{array}{l}\text { Rev Bras Psiq } \\
2: 31-39\end{array}$ \\
\hline 1983 & $\begin{array}{l}\text { Aspectos sociais, } \\
\text { culturais, políticos } \\
\text { e econômicos } \\
\text { relacionados ao } \\
\text { uso e abuso de } \\
\text { bebidas } \\
\text { alcoólicas no } \\
\text { Brasil }\end{array}$ & $\begin{array}{l}\text { JORGE } \\
\text { MR }\end{array}$ & $P S I Q$ & $\begin{array}{l}\text { Rev Assoc Bras } \\
\text { Psiq São Paulo } \\
5(18): 177- \\
178\end{array}$ \\
\hline 1987 & $\begin{array}{l}\text { Aspectos } \\
\text { psicossociais do } \\
\text { alcoolismo e a lei, } \\
\text { sob o prisma da } \\
\text { saúde pública }\end{array}$ & DIAS HP & SP & $\begin{array}{l}\text { CEBRID } \\
\text { Temas de } \\
\text { Saúde sob o } \\
\text { prisma da } \\
\text { Legislação } \\
\text { Sanitária } \\
\text { Brasileira } \\
\text { p.87-104 }\end{array}$ \\
\hline
\end{tabular}

* SP - Saúde Pública; PSIQ - Psiquiatria.

0 Quadro 1, referente à década de 1980, expõe apenas três publicações na área de alcoolismo. Mesmo não havendo produção científica de enfermeiros nas obras selecionadas, percebe-se a preocupação dos autores (médicos psiquiatras) em enfocar o uso, 0 abuso e a dependência alcoólica como um problema de saúde pública, considerando a importância conferida por eles às ações de prevenção e recuperação da saúde física e mental da comunidade.

Levando em consideração este enfoque, as estratégias de tratamento dos pacientes internados começaram a ser revisadas e contestadas. Os que até então eram tratados apenas em clínicas ou hospitais psiquiátricos, sob regime de internação, passaram a ser atendidos em clínicas particulares especializadas em álcool e outras drogas, ou nos hospitais públicos psiquiátricos, em setores especializados na doença. $\mathrm{A}$ partir daí, ênfase maior foi dada aos cuidados primários, à promoção e prevenção do alcoolismo, mostrando uma tendência a ver o problema não somente sob a ótica da Psiquiatria, mas também da Saúde Pública.

Outra questão importante foi o fato de os autores começarem a estudar o alcoolismo como uma doença multifatorial, ou seja, influenciada por fatores sociais, culturais, políticos e econômicos, além dos genéticos e familiares, concretizando a possibilidade de um olhar mais amplo para a solução dos problemas advindos do abuso de substâncias psicoativas, como no caso do álcool. A propósito, as novas tendências conceituais são no sentido de abandonar o termo alcoolismo pela sua conotação estritamente médica, que desconsidera a doença como multifatorial. 
Quadro 2- Produção científica sobre alcoolismo na década de 1990 - Rio de Janeiro, 2007

\begin{tabular}{|c|c|c|c|c|}
\hline $\begin{array}{c}\text { Ano de } \\
\text { Publicação }\end{array}$ & Título & Autor(es) & Abordagem* & Edição \\
\hline 1990 & $\begin{array}{l}\text { Prevención y } \\
\text { control del } \\
\text { abuso de } \\
\text { drogas }\end{array}$ & $\begin{array}{c}\text { GOSSOP } \\
\text { M.; GRANT } \\
\text { M. }\end{array}$ & $\mathrm{SP}$ & OMS \\
\hline 1991 & $\begin{array}{l}\text { Práticas } \\
\text { médicas, } \\
\text { toxicomanias e } \\
\text { a promoçãa } \\
\text { do exercício da } \\
\text { cidadania }\end{array}$ & CRUZ M.S. & $P S I Q$ & Dissertação \\
\hline 1993 & $\begin{array}{l}\text { Prevalência do } \\
\text { alcoolismo em } \\
\text { cuidado } \\
\text { primário } \\
\text { à saúde }\end{array}$ & $\begin{array}{c}\text { SAALFELD } \\
\text { V; } \\
\text { ALVARES- } \\
\text { DA-SILVA } \\
\text { MR }\end{array}$ & $\mathrm{SP}$ & $\begin{array}{l}\text { Rev Pesq } \\
\text { Méd } 27 \\
(1): 5-9\end{array}$ \\
\hline 1993 & $\begin{array}{l}\text { A prática de } \\
\text { enfermagem } \\
\text { psiquiátrica: } \\
\text { subordinação } \\
\text { e resistência }\end{array}$ & $\begin{array}{c}\text { FRAGA } \\
\text { MNO }\end{array}$ & $P S I Q$ & Livro \\
\hline 1995 & $\begin{array}{l}\text { As bebidas } \\
\text { alcoólicas e os } \\
\text { meios de } \\
\text { comunicação: } \\
\text { revisão de } \\
\text { literatura }\end{array}$ & $\begin{array}{l}\text { PINSKY J.; } \\
\text { SILVA MTA }\end{array}$ & $\mathrm{SP}$ & $\begin{array}{l}\text { Rev ABP- } \\
\text { APAL } 17 \\
\text { (3):115- } \\
121\end{array}$ \\
\hline
\end{tabular}

* SP - Saúde Pública; PSIQ - Psiquiatria.

0 Quadro 2 enfoca a década de 1990, época em que as publicações selecionadas abordaram a atenção especial que os profissionais da área da saúde deram ao atendimento ao alcoolista, à participação da família durante o seu tratamento e ao aprimoramento do processo de enfermagem psiquiátrica, com vistas à sua reabilitação, para que ele pudesse cumprir novamente suas funções familiares, sociais e econômicas.

Deve-se ressaltar que, nesta década, as cinco publicações selecionadas apresentaram diferentes abordagens, tendo sido escritas por psiquiatras, psicólogos e também enfermeiros, autores que começavam a descrever os distúrbios mentais e físicos dos pacientes, evidenciando a preocupação com o aumento da prevalência dos efeitos orgânicos, psicológicos e sociais causados pelo uso abusivo de álcool. Foi possível notar, nos referidos textos, a contestação do tratamento psiquiátrico dispensado ao paciente que abusava do álcool.

Esta contestação, que visava os modelos hospitalocêntrico e curativo de saúde vigentes, abriu espaço para o surgimento do modelo prevencionista. Com isso, a sociedade percebeu que o hábito de beber, até então considerado apenas uma questão cultural inofensiva, aprovada e estimulada há tempos, causava sérias conseqüências para quem fazia uso abusivo do álcool, com reflexos negativos na sua família, no seu trabalho e junto à comunidade.

Já é do conhecimento público que o uso crônico do álcool pode acarretar diversas alterações clínicas e psíquicas, com diferentes níveis de gravidade, devido à rapidez com que ele se distribui pelo organismo. ${ }^{7}$ Trata-se, portanto, de assunto que merece atenção não só dos profissionais da área de saúde, mas também dos terapeutas em álcool e drogas, bem como um plano de atendimento e reabilitação que minimize as seqüelas decorrentes em cada caso.

Quadro 3 - Produção científica sobre alcoolismo no período de 2000 a 2006 - Rio de Janeiro, 2007

\begin{tabular}{|c|c|c|c|c|}
\hline $\begin{array}{c}\text { Ano de } \\
\text { Publicação }\end{array}$ & Título & Autor(es) & Abordagem* & Edição \\
\hline 2001 & $\begin{array}{l}\text { Consulta de } \\
\text { enfermagem: } \\
\text { um } \\
\text { instrumento } \\
\text { na assistência } \\
\text { ao paciente } \\
\text { alcoolista }\end{array}$ & $\begin{array}{l}\text { RODRIGUES } \\
\text { LA; } \\
\text { BARBOSA } \\
\text { PPG; } \\
\text { OLIVIERA } \\
\text { SP; SOUZA } \\
\text { RG; } \\
\text { MACIEIRA } \\
\text { MS }\end{array}$ & SP & ABEAD \\
\hline 2001 & $\begin{array}{l}\text { Alternativas } \\
\text { para o } \\
\text { tratamento da } \\
\text { sindrome de } \\
\text { dependência } \\
\text { alcoólica } \\
\text { realizado por } \\
\text { enfermeiros }\end{array}$ & $\begin{array}{l}\text { OLIVEIRA } \\
\text { E; PILLON } \\
\text { SC }\end{array}$ & SP & $\begin{array}{l}\text { O Mundo da } \\
\text { Saúde } \\
\text { 3:285-294 }\end{array}$ \\
\hline 2001 & $\begin{array}{l}\text { Determinantes } \\
\text { socioculturais } \\
\text { do uso } \\
\text { abusivo de } \\
\text { álcool e } \\
\text { outras } \\
\text { drogas:uma } \\
\text { visão } \\
\text { panorâmica }\end{array}$ & $\begin{array}{c}\text { CRUZ MS; } \\
\text { FERREIRA } \\
\text { SMB }\end{array}$ & $\mathrm{SP} / \mathrm{PSIQ}$ & $\begin{array}{c}\text { Álcool e } \\
\text { drogas - uso, } \\
\text { dependência e } \\
\text { tratamento } \\
\text { (livro) }\end{array}$ \\
\hline 2002 & $\begin{array}{l}\text { O uso de } \\
\text { drogas } \\
\text { psicotrópicas } \\
\text { no Brasil }\end{array}$ & $\begin{array}{c}\text { MARLATT } \\
\text { BC }\end{array}$ & SP & $\begin{array}{c}\text { SENAD - } \\
\text { Formação de } \\
\text { Multiplicadores } \\
\text { de } \\
\text { Informações } \\
\text { Preventivas } \\
\text { sobre Drogas }\end{array}$ \\
\hline 2002 & $\begin{array}{l}\text { As drogas e } \\
\text { seus efeitos }\end{array}$ & SUCAR J.M & SP & $\begin{array}{c}\text { SENAD - } \\
\text { Formação de } \\
\text { Multiplicadores } \\
\text { de } \\
\text { Informações } \\
\text { Preventivas } \\
\text { sobre Drogas }\end{array}$ \\
\hline 2006 & $\begin{array}{l}\text { Prevenção no } \\
\text { local de } \\
\text { trabalho. } \\
\text { Conceitos } \\
\text { gerais, } \\
\text { avaliação } \\
\text { diagnóstica e } \\
\text { complicações } \\
\text { clínicas }\end{array}$ & $\begin{array}{l}\text { NIEL M; } \\
\text { JULIÃO AM }\end{array}$ & $\mathrm{SP} / \mathrm{PSIQ}$ & $\begin{array}{l}\text { Panorama } \\
\text { atual de } \\
\text { drogas e } \\
\text { dependência } \\
\text { (livro) }\end{array}$ \\
\hline
\end{tabular}

* SY - Saúde Pública; PSIQ - Psiquiatria. 
0 Quadro 3 enfoca os anos de 2000 a 2006, entretanto, nota-se um vácuo de publicações no período entre $2002 \mathrm{e}$ 2006. Observa-se, também, que as produções científicas elaboradas por psiquiatras, sociólogos e também por enfermeiros, mesclaram as abordagens em Psiquiatria e em Saúde Pública. Nesse período, surgem as políticas públicas em drogas, implantadas pelo Ministério da Saúde ${ }^{15} \mathrm{com} 0$ objetivo de reduzir o consumo alcoólico, coibir as internações hospitalares desnecessárias e, ainda, proteger e prover condiç̃̃es terapêuticas aos pacientes, internados ou não.

Estas ações apoiaram-se no modelo conceitual de saúde pública: agente, hospedeiro e ambiente. As ações do agente visavam controlar a qualidade e volume de produção, comercialização, distribuição e taxação; em relação ao hospedeiro, consumidor real ou potencial, as ações eram de caráter educativo e informativo visando uma possível mudança dos padrões de ingestão e de atitude em relação ao problema do alcoolismo e do alcoolista; quanto às ações sobre o ambiente e meio social, visavam correlacionar fatores sócio-culturais, hábito de beber e uso de medidas preventivas, na tentativa de resolução de conflitos gerados pela pobreza ou por causas psicológicas.

\section{CONSIDERAÇÕES FINAIS}

0 levantamento realizado pelas autoras teve como finalidade identificar o volume da produção científica sobre alcoolismo, sob o enfoque da Psiquiatria e/ou da Saúde Pública, produzida por profissionais da área da saúde. Constatou-se que, nos últimos 26 anos, foram nove as publicações com enfoque em Saúde Pública, três em Psiquiatria e dois voltados para ambas as áreas. Considerando que o alcoolismo é um grave problema de saúde pública, trata-se de uma produção incipiente, ainda mais porque em 2003 foi implantada a nova política do Ministério da Saúde para os problemas relacionados ao uso, abuso e dependência de álcool, o que deveria incentivar os pesquisadores da área de Saúde Pública a demonstrar maior interesse pelo tema.

É importante assinalar que a escassa produção de enfermagem abordando a temática está relacionada com 0 pouco conhecimento dos profissionais desta área a respeito de um assunto que, embora não seja novo, ainda gera um comportamento ambíguo no profissional que atende o usuário de álcool, o que certamente prejudica a sua atuação por não permitir que tenha uma percepção mais abrangente sobre a gravidade do problema.

Ressalta-se que, na atualidade, os cursos de graduação em Enfermagem já vêm fornecendo ao enfermeiro ensino teórico e prático de qualidade, tanto na área de Psiquiatria quanto na de Saúde Pública. Portanto, é de se esperar que a partir da sua atuação profissional junto ao alcoolista, ele possa aumentar a sua produção científica em função da proximidade com o problema.

Diante do exposto, as autoras consideram imprescindível que 0 enfermeiro busque novos saberes acerca do alcoolismo, o que lhe propiciará um novo olhar em relação ao uso abusivo de bebida alcoólica e a oportunidade de prestar uma assistência mais eficiente e eficaz a esta clientela específica.

\section{Referências}

1 - Davoli A, Mariano EC. Uso de bebidas alcoólicas por crianças: fenômeno a ser investigado. J Pediatr 1994; 70(1): 52-55.

2 - Saalfeld V, Alvares SMR. Prevalência do alcoolismo em cuidado primário à saúde. Rev Pesq Médica 1993 set; 27(1): 5-9.

3 - Dias HP. Aspectos psicossociais do alcoolismo e a lei sob o prisma da saúde pública. In: CEBRID, organizador. Temas de saúde sob o prisma da legislação sanitária brasileira. São Paulo (SP):1987.

4 - Donato M. Reinserção do trabalhador alcoolista no contexto laboral - a percepção do enfermeiro do trabalho. [tese de doutorado]. Rio de Janeiro (RJ): Escola de Enfermagem Anna Nery/ UFRJ; 2002.

5 - Galduróz JF, Noto AR. Uso pesado de álcool entre estudantes de $1^{\circ}$ e $2^{\circ}$ graus da Rede Pública de Ensino em dez capitais brasileiras. J Bras Dependência Química 2000 jan; 1 (1): 25-32.

6 - Oliveira E, Pillon SC. Alternativas para o tratamento da Síndrome de Dependência Alcoólica realizado por enfermeiros. 0 Mundo da Saúde 2001 jul/ set; 3 (25): 285-94.

7 - Niel M, Julião M. Conceitos gerais, avaliação diagnóstica e complicações químicas In: Moreira FGM, Silveira DX organizadores. Panorama atual das drogas e dependências. São Paulo (SP): Atheneu; 2006.

8 - Sucar JM. As Drogas e seus efeitos In: SENAD organizador. Formação de multiplicadores de informações preventivas sobre drogas. Brasília (DF); 2002.

9 - Masur J. Conjecturas sobre o uso milenar de bebidas alcoólicas. In: Jorge MR, organizador. Aspectos sociais, culturais, políticos e econômicos relacionados ao uso e abuso de bebidas alcoólicas no Brasil. Rev Associação Bras Psiquiatr 1983; 5(18): 177-78.

10 - Gorgulho M. A influência da mídia na realidade brasileira do fenômeno das substâncias psicoativas. In: Moreira FGM, Silveira DX, organizadores. Panorama atual das drogas e dependências. São Paulo (SP): Atheneu; 2006.

11 - Starkey PJ. Nurse's attitudes toward alcoholism. AORW J 1980; 31(5): 819-32.

12 - Sullivan EJ, Hale RE. Nurse's beliefs about the etiology and treatment of alcohol abuse: a national study. J Studf Alcohol 1987; 48(5): 456-60.

13 - Miranda CL. 0 parentesco imaginário: história e representação social da loucura nas relações do espaço asilar. São Paulo (SP)/Rio de Janeiro (RJ): Cortez/ UFRJ; 1994.

14 - Cruz MS. Formação de profissionais para o atendimento a usuário de drogas. [tese de doutorado]. Rio de Janeiro (RJ): Instituto de Psiquiatria/ UFRJ; 2001.

15 - Ministério da Saúde (BR). A política do Ministério da Saúde para a atenção integral a usuários de álcool e outras drogas. Secretaria Executiva - Coordenação Nacional de DST e AIDS. Brasília (DF); 2003. Série B - Textos Básicos de Saúde. 